\title{
Study of Melatonin as an Anti-Inflammatory Agent in a Rat Model of Perinatal Asphyxia
}

Luaces Juan Pablo ${ }^{1 *}$, Toro-Urrego Nicolas ${ }^{1}$, Merlo Sara ${ }^{2}$, Francisco Capani ${ }^{1}$

1. Instituto de Investigaciones Cardiológicas, Universidad de Buenos Aires, Consejo Nacional de Investigaciones Científicas y Técnicas, Buenos Aires, Argentina

2. Department of Biomedical and Biotechnological Sciences, Section of Pharmacology, University of Catania, Catania, Italy.

*Corresponding author: juanpluaces@yahoo.com

Perinatal asphyxia (PA) is a clinical condition characterized by a transient disruption of oxygen availability followed by metabolism deficits during birth. It was demonstrated that melatonin has neuroprotective effects throughout different mechanisms such as the reduction of oxidative stress, inflammation, and neurodegeneration. To evaluate the anti-inflammatory effect of melatonin in a PA model [1], in P7 rats the right common carotid artery (CCA) was isolated and permanently ligated in PA group $(n=13)$. After a $4 \mathrm{~h}$ recover period, animals were subjected to anoxia in a $100 \%$ nitrogen environment at $37^{\circ} \mathrm{C}$ for 3 minutes. In sham-operated group $(n=11)$ right CCA was exposed but not ligated, and no nitrogen was applied. One hour after anoxia, animals were injected intraperitoneally with vehicle solution $(n=10)$ or with $10 \mathrm{mg} / \mathrm{kg}$ of melatonin $(\mathrm{n}=12)$. At P8, animals were sacrificed and brains were analyzed either by immunohistochemistry or western blot (WB). Iba-1 immunoreactive cells were counted in the CA1 area of the hippocampus. Two slides per brain were analyzed and at least 8 counting frames were assessed per animal. PA rats injected with vehicle solution (7.72 Iba-1+ cells) presented a 1.42 fold increase in the number of Iba-1 positive cells, in comparison to sham rats treated with vehicle solution $(5.42 \mathrm{Iba}-1+$ cells $)(p<0.01)$, the protein level of Iba-1 also showed this increase by WB. PA animals treated with melatonin $(8.05$ Iba-1+ cells) showed no significative differences in the number of Iba-1 positive cells when compared to the PA group treated with vehicle. Protein level by WB showed a slight but non-significative decrease in the $\mathrm{PA}+$ melatonin group with respect to the PA group treated with vehicle. Further studies considering earlier stages of development are still necessary to evaluate the anti-inflammatory effect of melatonin for neuroprotective treatment following PA [2]. 

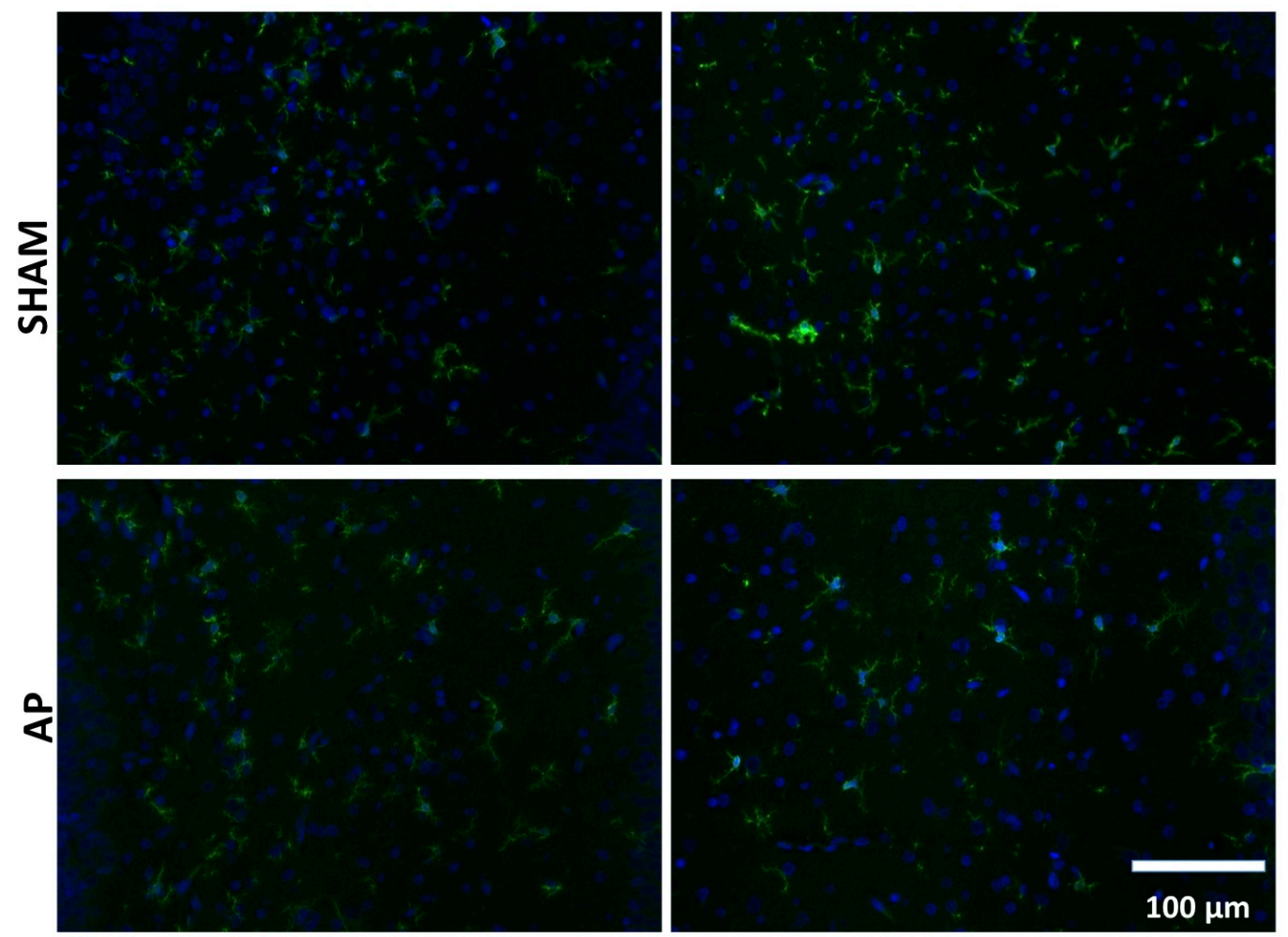

Vehicle

Melatonin

Figure 1. Representative photomicrographs of Iba-1 immunoreactive cells in the CA-1 region of the hippocampus in the different experimental groups. Inmunohistochemistry for Iba-1 (green) was counterstained with DAPI (blue).

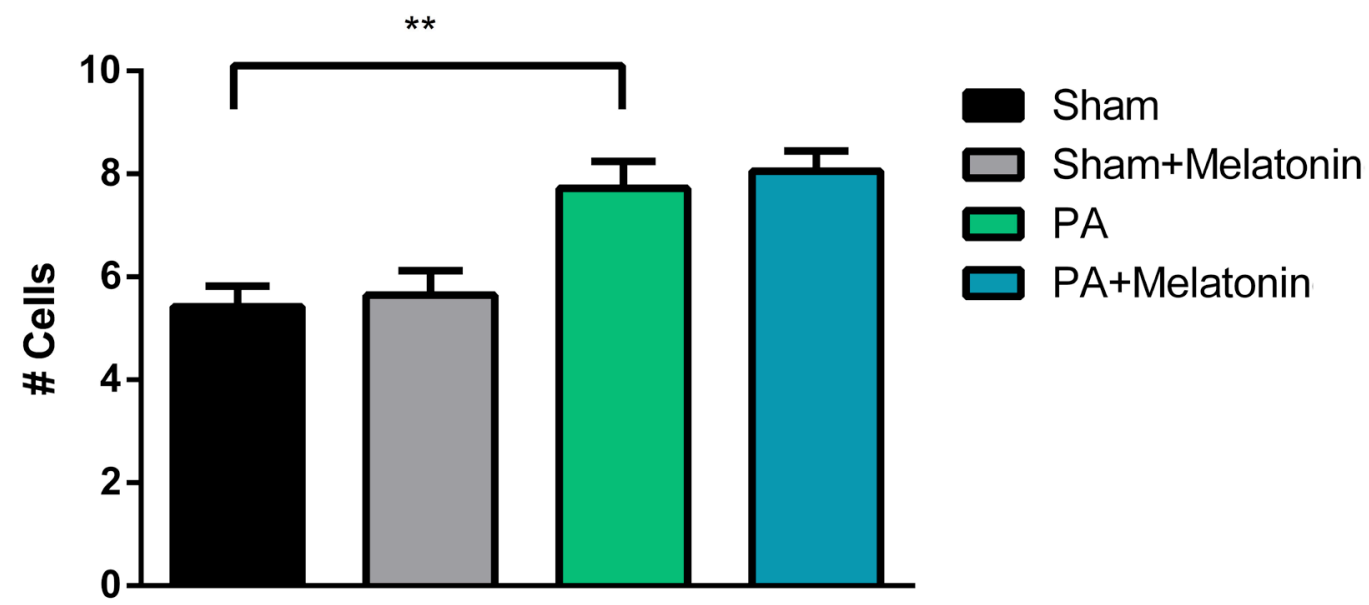

Figure 2. The histogram represents the number of the Iba-1 positive cells measured from each experimental group. Bars represent the mean + SEM. Student's $t$ test was employed to analyze the data $(* * \mathrm{p}<0.01)$.

References

[1] J.I. Romero, et al., Biochim. Biophys. Acta 1850 (2015), p. 1274.

[2] The authors acknowledge funding from the CONICET PIP 0779. 